\title{
Recherches en didactique des langues et des
} cultures

Les cahiers de l'Acedle

18-2 | 2021

Didactique des langues \& plurilinguisme(s) : 30 ans de recherches

\section{Pour une pédagogie de l'alternance des langues ou le plurilinguisme pour tous par l'intercompréhension}

Un aspect de « l'héritage » de Louise Dabène

\section{Christian Degache}

\section{OpenEdition}

Journals

Édition électronique

URL : https://journals.openedition.org/rdlc/9208

DOI : $10.4000 /$ rdlc. 9208

ISSN : $1958-5772$

Éditeur

ACEDLE

\section{Référence électronique}

Christian Degache, «Pour une pédagogie de l'alternance des langues ou le plurilinguisme pour tous par l'intercompréhension », Recherches en didactique des langues et des cultures [En ligne], 18-2 | 2021, mis en ligne le 30 septembre 2021, consulté le 11 octobre 2021. URL : http://journals.openedition.org/ rdlc/9208; DOI : https://doi.org/10.4000/rdlc.9208

Ce document a été généré automatiquement le 11 octobre 2021.

\section{cc) (†) $\Theta$}

Recherches en didactique des langues et des cultures is licensed under a Creative Commons AttributionNonCommercial-NoDerivatives 4.0 International License 


\title{
Pour une pédagogie de l'alternance des langues ou le plurilinguisme pour tous par l'intercompréhension
}

Un aspect de « l'héritage » de Louise Dabène

\author{
Christian Degache
}

\section{Introduction : autour d'une métaphore du bâtiment}

1 En 1995, trois ans après le lancement du projet Galatea fin 1991, Louise Dabène publie un article dans le numéro 98 des Études de linguistique appliquée sous le titre « Apprendre à comprendre une langue voisine, quelles conceptions curriculaires ?» (Dabène, 1995). Elle y posait la problématique de l'insertion curriculaire en s'interrogeant sur les stratégies de déploiement de l'intercompréhension en langues voisines, notamment pour la famille des langues romanes. Certes, 20 ans auparavant, Louise Dabène avait publié «L'enseignement de l'espagnol aux francophones: pour une didactique des langues voisines ", dans le numéro 39 de la revue Langages (Dabène, 1975) et aussi, en 1984, « Langues voisines... langues faciles? » dans le numéro 113 de la revue Les Amis de Sèvres sur la thématique de La latinité aujourd'hui (Dabène, 1984) qui recueillait les communications présentées lors d'un colloque au CIEP de Sèvres. Mais dans ces deux cas, sans le mot intercompréhension, qui apparaitra seulement sous sa plume en 1991 dans la candidature du projet Galatea (1 $1^{\text {ère }}$ phase ${ }^{1}$ ) et en espagnol dans « Aprendizaje de la intercomprensión entre locutores de lenguas románicas », dans les actes du Congrès international d'espagnol langue étrangère de Grenade (Dabène, 1992). En revanche, le mot était bien présent dans la communication présentée par Francis Debyser « Peut-il y avoir une didactique de l'intercompréhension des langues romanes? » à l'occasion du même colloque La latinité aujourd'hui (Debyser, 1984) au CIEP de Sèvres et dans l'objectif même de la rencontre qui était d'apporter " des éléments de réflexion à tous ceux qui considèrent l'intercompréhension latine comme un facteur de solidarité entre des peuples partageant le sentiment d'appartenance à une civilisation, à une aire 
linguistique communes» (Feneuille, 1984). De ce colloque, qui s'est tenu du 8 au 10 mars 1983, et auquel Bernard Py participait également (Py, 1984), Louise Dabène disait qu'il avait joué un rôle déterminant dans son parcours. Il est vrai que les organisateurs, faisant le constat de l'«impression de familiarité » ressentie par tout voyageur de langue romane circulant dans "les pays de la latinité », y posaient une question qui faisait directement écho à son article de 1975 et qui serait l'objet de nombreux développements dans les décennies à venir : «Peut-on s'appuyer sur cette "familiarité" pour améliorer et enrichir l'enseignement d'une langue romane à des apprenants romanophones pour développer éventuellement en eux l'aptitude à comprendre une troisième langue de la même famille?» (Feneuille, op. cit.)

2 La publication en 1995 dans les ELA de l'article que nous mettons ici en exergue, correspond ainsi à un moment-clé de la trajectoire de Louise Dabène sur la notion d'intercompréhension (IC). Ce moment se situe presque au terme de la phase dite " prédidactique » du projet Galatea (étude préalable avant la réalisation) qui poursuit deux objectifs : (1) recueillir et analyser les stratégies et représentations d'échantillons représentatifs en lien avec l'expérience de la parenté des langues romanes, et (2) mener à bien une analyse contrastive de ces langues dans la perspective d'identifier des "règles de passage et de vigilance ». Le tout en vue de la mise en œuvre d'une $2^{\text {ème }}$ phase consistant à l'«élaboration d'un guidage méthodologique adapté " (Dabène, 1994 : 42), entendue comme la phase didactique du programme centrée sur la création de matériel pédagogique. Celle-ci viendrait juste après, à partir de décembre 1995, avec l'approbation du projet de réalisation de cédéroms (également nommé Galatea) par l'Union européenne dans le cadre du programme Socrates/Lingua (Dabène et al., 2001). Le principe fondateur de ces travaux est bien sûr de reconsidérer positivement le rôle accordé à la parenté linguistique dans l'enseignement/apprentissage des langues voisines, c'est-à-dire d'en faire un véritable atout pour l'apprentissage. Un principe dans l'air du temps, d'une part parce que l'idée courait depuis déjà quelques années, comme nous l'avons vu (Dabène, 1975, 1984 ; Debyser, 1984), d'autre part parce que d'autres projets émergeaient contemporainement au début des années 90 , notamment EuRom4 (Blanche-Benveniste 1991, 1992; Llamas Pombo \& Uzcanga Vivar, 1992), Intercommunicabilité romane (Schmitt Jensen, 1997), EuroCom (Klein \& Stegmann, 2000).

3 Dans cet article sur les conceptions curriculaires de 1995, Louise Dabène file la métaphore du bâtiment. Ainsi parle-t-elle tour à tour d'«état des lieux", puis de "lézardes de l'édifice » (p. 105-106) pour dégager quatre évolutions en cours et leurs conséquences dans l'appropriation et l'enseignement des langues. D'abord (1) le fait que l'orientation générale de l'appropriation se caractérise par davantage d'acquisition informelle et une diversification des situations d'appropriation linguistique. Elle pointe ensuite (2) une évolution du "statut fonctionnel de la langue enseignée ", à savoir que celle-ci de plus en plus passe du statut de «langue objet » à celui de " langue medium » des apprentissages. Une autre évolution tient pour elle (3) au développement de l'enseignement aux adultes, induisant de nouveaux besoins et, partant, la nécessité de définir de nouveaux objectifs, moins «maximalistes", "plus ciblés et plus hiérarchisés ", notamment des "traitements pédagogiques dissociés » des habiletés, surtout parce que ne sont pas mis "en jeu les mêmes activités cognitivo-langagières » (ex: stratégies d'inférence et élaboration dominantes en réception). Enfin, (4) elle souligne la nécessité grandissante de prendre en compte les acquis antérieurs de 
l'apprenant, notamment sa perception de la proximité/distance de la (des) langue(s) cible et «la gestion de son itinéraire personnel d'apprentissage en fonction de phénomènes bien connus comme le décalage entre compréhension et expression mais aussi du degré de confiance que donne la représentation de la proximité ». Quatre constatations encore plus vraies aujourd'hui, de manière accrue ou renouvelée, notamment pour la quatrième.

Louise Dabène présentait ensuite six " principes directeurs d'un schéma curriculaire » (p. 107-110) :

- «Dissociation provisoire des compétences »: ces dernières étant entendues comme les habiletés langagières, avec l'idée de prioriser les habiletés de réception.

- «Étayage par les savoirs antérieurs » : mobiliser et intégrer tout type de savoir linguistique, discursif et extralinguistique (sur le monde), pour faire flèche de tout bois dans les activités de compréhension.

- «Incitation à l'actualisation du potentiel cognitif»: amener l'apprenant à prendre conscience de ses moyens et stratégies et à les développer, en confiance.

- «Sélection et hiérarchisation de l'information métalinguistique » : une attitude confiante en réception se gagne par une démarche réflexive conduisant à la connaissance de « règles de passage » et « de vigilance».

- «Contextualisation et construction progressive de l'information» : se construire au fur et à mesure des activités réceptives une grammaire contrastive et plurilingue de la compréhension.

- «Conception interactive du travail et développement de l'autonomie » : le développement des compétences doit se réaliser à travers la réalisation de tâches significatives dans lesquelles l'apprenant s'implique réellement.

Ces six principes directeurs ont servi à l'élaboration de matériel pédagogique (les cédéroms Galatea), la construction de modules (sur Galanet), de cours, de formation d'enseignants, moins directement, toutefois, à la conception ou à l'insertion curriculaire. Deux orientations semblent en revanche y contribuer davantage à la page 110 :

- «Apprentissage "répercuté" »: l'étude ou la découverte d'une langue romane «peut permettre à l'apprenant de rebondir sur la découverte d'une troisième langue romane »; ainsi pour Louise Dabène (p. 110) "Diverses formules d'apprentissage "répercuté" pourraient être imaginées dans cette perspective ».

- «Déroulement curriculaire »: Louise Dabène en vient à suggérer une progression en 3 étapes :

- mobilisation du potentiel cognitif/des savoirs latents à partir de textes et discours familiers ;

- élargissement à d'autres modèles textuels et discursifs ;

- utilisation des acquis langagiers pour la communication et pour l'étude de «contenus nouveaux » : les langues objet de compréhension deviennent langues médium.

Deux orientations curriculaires qui ont connu depuis de multiples répercussions suggérées par Louise Dabène en conclusion : « imaginer des organisations curriculaires fondées sur des structures modulaires accordant la priorité, mais non l'exclusivité, au développement de telle ou telle compétence pendant des durées limitées ». Lesquelles ? Que s'est-il fait en ce sens depuis 25 ans? C'est ce que nous nous proposons de développer ci-après en montrant que l'insertion curriculaire de l'intercompréhension s'est développée selon différents axes qui permettent de dessiner sept types de scénarios. 


\section{Sept scénarios d'insertion curriculaire de l'intercompréhension}

5 Clarifions d'entrée que, on l'aura compris, tous les développements de l'intercompréhension ne sont pas directement reliables aux suggestions de Louise Dabène. À travers ses différents travaux, elle a néanmoins inspiré d'une façon ou d'une autre la plupart de ces scénarios d'intégration. Elle a en tout cas été concernée par un grand nombre d'initiatives en France et en Europe, mais aussi en Amérique Latine. Louise Dabène associait en effet ce continent à ses travaux sur ce sujet dès leur lancement au début des années 90: Patricio Moreno et Gilda Tassara de l'équipe chilienne de Linguasur, respectivement des universités de Concepción (UDEC) et de Valparaiso (UPLA) qui ont produit ensuite la méthode Interlat (Tassara et al., 2007; Villalón et al., 2011) ; Ana Carullo de la Universidad Nacional de Córdoba en Argentine qui a lancé le projet InterRom, lequel a également donné lieu à la publication d'une méthode éponyme (Carullo et al., 2007, Carullo \& Torre, 2009 ; Carullo \& Marchiaro, chap 3 in Degache \& Garbarino, 2017) puis Maria Helena Ceberio de la Universidad Nacional de Rio Cuarto, laquelle, après un séjour de recherche au Lidilem au temps du projet Galatea, a participé activement aux sessions Galanet et à la diffusion de l'intercompréhension interactive en Argentine et en Amérique latine (Ceberio, 2011).

6 Louise Dabène avait un attachement très fort à ce continent, en tant qu'hispaniste bien sûr, et par son fils Olivier Dabène, professeur de science politique à l'Institut d'Études Politiques de Paris et Président de l'Observatoire Politique de l'Amérique Latine et des Caraïbes, qui en a fait son champ d'études (voir notamment 0 . Dabène, 2020, et autres publications ${ }^{2}$ ).

7 Concernant le Brésil, Louise Dabène s'était impliquée dans le projet bivalence (didactique intégrée du portugais langue maternelle et du français langue étrangère ; Chaves da Cunha, 2001) et avait participé aux Sedifrales à Rio de Janeiro. Elle a aussi coordonné avec son mari Michel un projet CAPES-Cofecub de partenariat francobrésilien avec l'UNICAMP (Campinas - SP), de 1997 à 2001, sur la lecturecompréhension en contexte universitaire (LM \& LE) qui a donné lieu à plusieurs publications marquantes (voir notamment Serrani Infante, 2001).

8 Il n'y a pas lieu de s'étonner, par conséquent, que, compte tenu des pistes qu'elle a tracées, l'héritage de Louise Dabène se retrouve, associé à celui des autres précurseurs des années 90 que nous avons cités plus haut, dans de nombreuses initiatives en faveur de l'insertion curriculaire de l'intercompréhension. Celle-ci se retrouve à notre sens, dans plusieurs types d'intégration que nous essayons de caractériser depuis quelques années en posant comme point nodal la fonction qu'y joue l'intercompréhension (Degache et al., 2012 ; Degache \& Garbarino, 2017 : 19). Nous y revenons ci-après autour de sept types que nous proposons de désigner comme des scénarios, en attribuant à ce terme toute sa polysémie italienne ${ }^{3}$ recouvrant à la fois les éléments scéniques qui représentent le cadre de l'action et le canevas de cette action, son déroulement.

\section{L'intercompréhension propédeutique}

Dans ce scénario, l'intercompréhension assure une fonction propédeutique, au sens étymologique du terme, d'«enseigner auparavant». Avant de commencer 
l'apprentissage d'une langue-cible qui entretient des liens de parenté avec une/des langue(s) du répertoire langagier des apprenants, on amène les apprenants à identifier ces liens, à les questionner, à essayer de les expliquer et dégager quelques régularités. Comme l'écrit Blanchet (2018: 32), « faire prendre conscience que cette langue est déjà en partie compréhensible, que l'on a une compétence de réception relativement développée, c'est identifier les ressources à partir desquelles on pourra plus facilement développer une compétence de production ».

10 Bien des manuels de FLE, E/LE, P/LE, notamment dans les versions pour publics spécifiques (exemple en FLE pour des apprenants lusophones: Alter Ego + pour le Brésil, Hachette/Aliança Francesa ${ }^{4}$ ), mentionnent l'intérêt de la parenté linguistique pour l'apprentissage dans les premières unités didactiques (souvent dans un « dossier $0 »)$. Mais la contrastivité est centrée ensuite rapidement sur les questions socioculturelles, bien peu sur les aspects linguistiques et cognitifs, ni socio-affectifs, lesquels sont en définitive laissés à l'initiative de l'enseignant. Il y a tout lieu de penser que cette propédeutique pourrait être plus marquée et ne pas se limiter à deux langues car le répertoire et les intérêts des apprenants sont plus larges. Par exemple, au Brésil, tout apprenant débutant en français a dans son répertoire au moins quelques notions d'espagnol, voire quelques mots en italien, mais surtout un vocabulaire anglais apparenté au français (danger, choice, age, budget...) sans équivalent en portugais.

\section{L'intercompréhension médiatrice}

11 Sur des bases proches de l'IC propédeutique, ce scénario donne à l'intercompréhension une fonction de médiation en suscitant la réflexion métalinguistique entre une - ou plusieurs - langue(s) apparentée(s) et la langue de scolarisation (L1 ou langue maternelle). La comparaison inter-linguistique, en stimulant analogie, transfert, et élaboration de règles de correspondance entre les langues doit bénéficier en premier lieu à la connaissance de la langue 1. Le premier objectif est ici le développement de la conscience métalinguistique, chère à Louise Dabène (1992 ; Dabène \& Ingelmann, 1996 : 22) et au cœur de l'Éveil aux langues. D'ailleurs, la plupart des programmes d'Éveil aux langues/Language awareness ont compté dès leur origine des activités et modules s'inscrivant dans cette perspective, à commencer par ceux proposés par Eric Hawkins (1984). Ainsi, le $8^{\text {ème }}$ fascicule de la série Awareness of Language, réalisé par Jim McGurn (1991), s'intitule-t-il «Comparing Languages» et a pour objectif de montrer que les langues européennes ne sont pas si «étrangères ». Comparer est en effet une "stratégie cognitive de traitement» essentielle selon Bégin (2008: 48) qui ne figure pourtant " habituellement pas dans les taxonomies " alors qu'il s'agit d'une stratégie « tout aussi importante pour la réalisation de tâches impliquant notamment l'analyse et la résolution de problème» (ibid., p.58), comme le démontre le DVD «Comparons nos langues ${ }^{5} »$ (Auger, 2004) pour les élèves des classes d'accueil et qui va bien au-delà des langues européennes et apparentées (Auger, 2007 ; voir aussi l'analyse de Alaoui, 2007). La plupart des projets d' «Éveil aux langues » comptent toutefois un ou plusieurs volets autour de la parenté linguistique, bien souvent à partir de la famille des langues romanes.

12 Le colloque DILAPLU fut l'occasion de découvrir un exemple caractéristique de ce scénario avec la présentation de Chazalon et\& al. qui proposent à des élèves de 7-8 ans d'une classe française de CE1 un "détour» par des énoncés compréhensibles en 
espagnol, langue inconnue d'eux, pour apprendre à identifier le verbe en français grâce à la comparaison des structures négatives (Chazalon et al., ici-même). L'intercompréhension, à savoir ici la possibilité de comprendre des énoncés sélectionnés en langue(s) cible(s) et de les mettre en contraste avec la langue de scolarisation, joue un rôle de déclencheur d'intérêt, de catalyseur d'attention. À cet effet, de multiples langues cibles peuvent être utilisées, y compris des langues modimes (moins diffusées et moins enseignées) comme les créoles ou des langues régionales.

Ce scénario est pratiqué dans des écoles au Brésil, notamment dans l'état de Rio Grande do Norte sous la direction de Selma Alas Martins (2014), avec des enfants de 10-11 ans, ou encore avec des collégiens de 13-15 ans (Souza, 2013), à partir de la découverte écrite et orale de divers genres textuels dans plusieurs langues romanes dont l'espagnol, le français et l'italien. Les résultats obtenus montrent les retombées positives sur la maitrise de la langue de scolarisation (le portugais en l'occurrence), la compétence discursive et la motivation, voire sur l'estime de soi (Sarsur, 2020), y compris bien entendu pour entreprendre un apprentissage plus durable et approfondi de ces langues, ou tout du moins de l'une d'entre elles. Cette démarche fait l'objet au Brésil, à l'heure actuelle, de plusieurs initiatives de recherche-action du même ordre, que le projet DIPROlinguas ${ }^{6}$ a entrepris de fédérer depuis début 2018. Ce projet, qui réunit 10 partenaires universitaires ( 8 au Brésil coordonnés par l'UFMG, 2 en France coordonnés par l'UGA), a précisément pour finalité d'étudier le potentiel de la réflexion comparative à partir de la perception des relations de distance et proximité entre les langues-cultures impliquées ou présentes dans l'environnement de l'apprenant au Brésil et en France ${ }^{7}$. Il donne également lieu à des actions de formation et recherche en direction des enseignants comme la formation ELMIC (Ensino de Línguas em Minas para e pela InterCompreensão $\left.0^{8}\right)$ avec deux objectifs principaux : (1) renforcer l'apprentissage du portugais langue de scolarité par une approche intercompréhensive qui conduit à la réflexion sur les langues; (2) éveiller et stimuler l'intérêt des élèves des écoles publiques pour l'apprentissage des langues étrangères au collège et au lycée. Il s'agit notamment de mobiliser les enseignants de portugais langue maternelle disposant d'une habilitation en français ou italien, acquise durant leurs études universitaires. Deux formations semblables sont à l'étude dans les états de São Paulo et du Paraná où l'accent est mis en particulier sur l'enseignement du portugais langue de scolarisation à des publics issus de l'immigration, dont une part importante compte au moins une langue romane dans leur répertoire (principalement espagnol, créole haïtien et français).

\section{L'intercompréhension instrumentale}

14 Ce scénario d'intégration de l'IC, sans aucun doute le plus connu, correspond au « déroulement curriculaire » en trois étapes présenté par Louise Dabène (1995) dont nous avons parlé plus haut. L'objectif est de comprendre des textes authentiques dans des langues jamais étudiées auparavant. L'IC y est dotée d'une fonction instrumentale à double titre :

- instrument des apprentissages linguistiques : découvrir la parenté, les règles de passage (par exemple avec les 7 tamis de EuroComRom, Meissner et al., 2004) et les règles de vigilance interlinguistiques;

- instrument des apprentissages extralinguistiques, c'est-à-dire avec des objectifs spécifiques, à partir de textes et discours d'un domaine de spécialité. 
Cette fonction instrumentale est chronologiquement la première à avoir généré son propre matériel pédagogique autour de la compréhension écrite : EuRom4 en 1997 (puis EuRom 5, Bonvino et al., 2011), Galatea (à partir de $2000^{\circ}$ ), puis les déjà cités EuroCom, InterRom, InterLat. Des matériels qui se limitent à la première fonction instrumentale ci-dessus, le plus souvent avec des textes de presse et sans objectifs spécifiques, autrement dit des «textes et discours familiers » conformément à la 1 ère étape définie par Louise Dabène. Les langues n'y sont pas hiérarchisées, c'est-à-dire qu'elles sont présentées conjointement, en "bouquet", même si leur abord a lieu consécutivement lors de la même séance ou sur une brève période de temps.

Ce n'est que plus tard que se développent des travaux liés aux deux autres étapes et à la deuxième fonction instrumentale ci-dessus, en lien avec un besoin localisé comme l'épreuve de "proficiência » en langue étrangère au Brésil qui conditionne l'admission en master et doctorat à la validation de la compétence à lire et comprendre des textes de son domaine de spécialité en langue étrangère (voir Albuquerque-Costa \& Miranda de Paulo, 2012 et dans Degache \& Garbarino, 2017 : 99 et chap. 8). Une démarche ensuite développée et perfectionnée par Lívia Miranda de Paulo dans sa thèse de doctorat (2018) pour former de futurs enseignants-chercheurs en didactique des langues à partir de la lecture de textes académiques de ce domaine de spécialité en version originale.

Le projet LALIC (Lecturas sobre América Latina en InterComprensión) piloté par Laura Masello en Uruguay (2018, Díaz \& Campanella, 2019) constitue un exemple récent de la mise en œuvre de cette fonction, dans l'optique de faire connaitre à des étudiants de sciences humaines «des œuvres sur la pensée critique latino-américaine écrites en plusieurs langues du continent (espagnol, français et portugais)» (Masello, $2018: 116$ ), "proches des études postcoloniales: Walter Mignolo (2007) en Argentine, Silviano Santiago (2000) au Brésil, Édouard Glissant $(1996,1997)$ en Martinique » (ibid. : 113).

\section{L'intercompréhension intégrée}

Par la fonction intégrative, il s'agit d'intégrer des enseignements disciplinaires autour d'un groupe de langues de la même famille. Ainsi va-t-on étudier une matière (sciences, histoire, mathématique, technologie, géographie, théâtre...) à partir de documents en différentes langues apparentées. À la différence de la fonction instrumentale, la discipline (non-linguistique) et ses contenus passent avant les textes, discours et documents: les objectifs prioritaires sont les connaissances et savoir-faire de la discipline et non pas les compétences langagières. Même si celles-ci ne sont pas négligées, elles sont avant tout le médium de l'apprentissage des contenus notionnels. L'intercompréhension intégrée est ainsi à l'intercompréhension ce que CLIL (Content and Language Integrated Learning) ou EMILE (Enseignement d'une matière par l'intégration d'une langue étrangère) est à l'enseignement d'une langue en particulier. Le matériel qui représente par excellence cette fonction est la méthode Euro-Mania (Escudé, 2008 ; voir aussi Escudé, 2012 ; Domingues Fonseca, 2017), « le seul moyen pédagogique à avoir été conçu selon les principes de l'intercompréhension intégrée » (Fonseca, chap 2: 47 in Degache \& Garbarino, 2017) pour un public-cible de 8 à 12 ans (fin école primaire et début du collège). Citons toutefois Carullo et Marchiaro (chap 3 in Degache \& Garbarino, 2017) qui font état de l'élaboration d'un matériel qui s'articule autour de textes en lien avec des thématiques abordées dans les disciplines du secondaire en Argentine. 
19 Si l'on compare l'intercompréhension intégrée avec le "déroulement curriculaire " proposé par Louise Dabène en 1995, on se rend compte que dans Euro-mania on commence directement à l'étape 3, les étapes antérieures y étant "intégrées » (surtout l'étape 1 car les "documents" utilisés pour étudier les thèmes abordés dans les matières sont des documents familiers en contexte scolaire) en tant qu'étapes finales de chaque module/fascicule sous le nom « Voyageons dans nos langues » (étape 4/5) et «Famille de langues» (étape 5).

\section{L'intercompréhension immersive}

20 Comme l'immersion en langue-cible, il y a l'immersion en intercompréhension. C'est sa fonction immersive. De quoi s'agit-il? Il n'existe pas a priori de «pays de l'intercompréhension", mais il existe de nombreux contextes favorables aux interactions intercompréhensives: zones frontalières, espaces internationaux académiques, professionnels, sportifs..., où cette immersion est possible car elle est acceptée ou acceptable. La notion d'acceptabilité, selon laquelle il faut nécessairement poser qu'« on va réussir à s'entre-comprendre [pour] mobiliser des ressources, des vigilances, des aides, des régulations", et "réussir à produire au moins un peu d'intercompréhension " (Blanchet, 2018: 32), s'avère en effet essentielle dans cette immersion. Si bien qu'il est possible de créer des contextes présentiels (Capucho, chap 13; Deransart et al., chap 14 in Degache \& Garbarino, 2017) ou distanciels, où l'intercompréhension interactive fait partie du contrat d'usage: chacun peut s'exprimer dans sa (ou ses) langue(s) romane(s) de référence et s'emploie à se faire comprendre et à comprendre les autres. Cette intercompréhension immersive peut être précédée d'une phase instrumentale ou être proposée d'emblée comme c'est le cas en master à l'Université de Grenoble ou encore à l'Université de Lyon, laquelle a créé un «parcours pluriannuel» (Garbarino, 2019: 40) sur deux années/quatre semestres qui commence par une telle immersion en ligne (ibid. : 46-47), principalement basée sur l'interaction écrite (forums, chats, messagerie), et qui trouve un écho au semestre 3 avec l'interaction orale (ibid. : 52).

21 Pratiquée depuis 2004 successivement sur les plateformes Galanet, Galapro et Miriadi, il s'agit d'immersion en ce sens que les exigences actionnelles de l'interaction internationale et plurilingue, tournée vers la télécollaboration, et donc la réalisation de productions finales (la «tâche » de la perspective actionnelle), s'imposent à n'importe quelle autre nécessité langagière, culturelle, métacognitive ou didactique. Un constat répété au fil des multiples sessions organisées sur ces plateformes (Degache, 2018) est celui que la compréhension mutuelle en plusieurs langues dans un tel cadre télécollaboratif, se révèle facteur de motivation, d'envie de découvrir et d'apprendre, comme c'est notamment le cas dans les sessions Romanofonia e cinema sur Miriadi (Degache \& Silva, 2020 ; Da Silva \& Degache, sous presse).

22 L'IC immersive trouve naturellement sa place en étape $3 \mathrm{du}$ «déroulement curriculaire " proposé par Louise Dabène en 1995, dans la mesure où on utilise les acquis langagiers pour la communication. Rien n'empêche pour autant, comme nous l'avons vu, de commencer directement par cette immersion dès lors que cette interaction est acceptée par les participants - parce que leur répertoire langagier, leur vécu et leurs représentations le leur permettent - et/ou rendue acceptable grâce aux 
moyens et modalités mis en œuvre pour une immersion intensive dans des pratiques d'alternances codiques.

\section{L'intercompréhension répercutée}

Nous reprenons là l'adjectif "répercuté » utilisé par Louise Dabène en 1995 en lui conférant un sens plus restreint. C'est sans doute le scénario le plus difficile à définir, le plus "contre nature» car sa problématique est la suivante: comment renforcer l'apprentissage d'une langue en exposant les apprenants à d'autres langues, donc, en substance, en lui prenant du temps?

Il s'agit de soutenir et renforcer l'enseignement/apprentissage d'une langue cible en particulier en cherchant des effets de répercussion sur et à partir d'autres langues, et ce, en les hiérarchisant. Cela passe par un scénario précis et un contrat codique et d'apprentissage plus exigeant. Par exemple, dans un cours de portugais de niveau B1 ou supérieur dans une université francophone : le portugais est ciblé prioritairement, les productions écrites et orales sont faites en portugais, les interactions en classe aussi, c'est la langue véhiculaire. L'usage du français est aussi réduit que possible conformément aux dispositions d'une approche communicative et actionnelle. Mais les apprenants sont amenés à lire et écouter les autres langues romanes (espagnol, catalan, italien), à interagir en ligne avec des locuteurs s'exprimant dans ces langues et à leur répondre en français (exceptionnellement en portugais). Non seulement ils doivent apprendre à comprendre ces autres langues mais ils doivent aussi apprendre à parler en classe de ces langues et de leurs relations avec le français et le portugais, de les comparer, en s'exprimant en portugais langue 2 .

La fonction répercussive de l'IC appliquée à l'apprentissage d'une langue cible spécifique (langue romane 2: LR2, cf. Carrasco et al., 2008), en mettant celle-ci en perspective avec d'autres de la même famille, provoque des effets de contraste et transfert (les 'répercussions'), mais aussi et surtout une motivation communicative authentique. Certes, le contrat codique est plus contraignant puisqu'il s'agit en quelque sorte de combiner l'intercompréhension immersive avec des productions en langue cible. C'est ce qui était pratiqué de 2016 à 2018 à l'Université Fédérale de Minas Gerais dans le cadre d'un cours d' "Estudos Temáticos da Língua Francesa»: un groupe d'étudiants brésiliens (portugais LR1) de français (LR2, niveau B1) a participé à trois reprises à une formation télécollaborative "Romanofonia e cinema » sur la plateforme Miriadi : production et interaction devaient se faire principalement en portugais sur la plateforme selon la consigne "vous offrez votre langue aux autres qui, en échange, vous offrent la leur ». En revanche, dans l'équipe locale, en classe ou ailleurs, les échanges et les productions orale et écrite devaient se faire en français.

Le grand avantage de la fonction répercussive de l'IC c'est d'abord que l'équipe locale n'est jamais en panne de sujet de communication. Les thèmes, les tâches, les nécessités et les événements de la session plurilingue en ligne, vont alimenter les échanges et productions langagières internes à l'équipe locale. Elle va surtout donner l'occasion d'exercer l'activité métalinguistique et métacognitive en LR2 ainsi que l'alternance codique finalisée, toutes choses de grande nécessité dans une compétence plurilingue. 


\section{L'intercompréhension didactique}

La $7^{\text {ème }}$ et dernière fonction d'intégration de l'IC que nous évoquons ici est la fonction didactique. Pratiquer l'intercompréhension est en effet un excellent vecteur de formation à la didactique des langues en général et à la didactique du plurilinguisme en particulier. Ainsi voit-on apparaitre des leitmotiv tels que se former à l'intercompréhension par l'intercompréhension (cf. la formation ELMIC citée plus haut). C'est aussi précisément l'objet des sessions sur la plateforme Galapro (Araújo e Sá et al., 2009, 2010 ; De Carlo \& Hidalgo Downing, chap 10 in Degache \& Garbarino, 2017 ; Degache, 2018), aujourd'hui substituées par des sessions spécifiques sur Miriadi, telle que la session Scuola_IC qui a eu lieu en $2019^{10}$. Il y est notamment question de découvrir et analyser des matériels et réalisations existants, de connaitre les problématiques de l'IC, d'en élaborer de nouvelles, d'apprendre à concevoir des séquences didactiques pour l'IC, pour différents publics dans diverses situations et, ce faisant, de découvrir et pratiquer les référentiels des approches plurielles et de l'intercompréhension (De Carlo \& Anquetil, 2019; Hidalgo Downing, 2019) et d'alimenter la Base d'Activités de l'Intercompréhension (BAI). ${ }^{11}$

Il est également possible de poursuivre ces objectifs didactiques dans une session hybride réunissant des publics suivant un scénario immersif et/ou répercuté comme c'est le cas dans la session en cours en 2020, Romanofonia e Cinema 4: Bambini partigiani, infancias clandestinas ${ }^{12}$ où trois des 12 équipes locales (à Barcelone, Grenoble et Lyon) réunissent des étudiants de master de didactique des langues. Il leur est possible d'opter pour une tâche de nature didactique (conception d'une séquence pédagogique par exemple) et de former des groupes de travail élargis à d'autres participants pour aller dans ce sens.

\section{Une pédagogie de l'alternance des langues}

Les sept fonctions que peut assumer l'intercompréhension, au cœur de ces sept scénarios, peuvent se combiner, comme nous l'avons suggéré au fil de leur présentation. Une formation peut actualiser en même temps deux fonctions pour le même public, ou une session d'intercompréhension en ligne peut, en se dirigeant à différents publics, laisser place à différentes fonctions.

Un point à discuter est celui de la désignation générale d'une telle pédagogie. Une première option serait de la désigner comme une pédagogie de l'alternance codique. L'inconvénient de cette désignation est qu'elle est centrée sur le code. Ce faisant, elle tend à offusquer l'alternance entre locuteurs et peut laisser croire que l'on va enseigner à changer de langue avec une intention, de manière à remplir une des fonctions de l'alternance codique (Dabène \& Billiez, 1988; Ali-Bencherif, 2009). Choisir la désignation de pédagogie du contact des langues, sans être erroné, serait pour sa part réducteur. En réalité on veut aller plus loin que le simple contact, vers des pratiques interactives d'alternance. C'est pourquoi notre choix se porte sur pédagogie de l'alternance des langues. En 2001, déjà, Danièle Moore posait la question: "une didactique de l'alternance pour mieux apprendre?». Il nous semble que cette désignation présente l'avantage de garder toute sa polysémie puisqu'elle recouvre l'alternance des langues lues ou écoutées (en réception écrite et/ou orale); l'alternance, dans l'interaction, des langues utilisées par les interlocuteurs; 
l'alternance dans les documents produits, souvent très créatifs, qui rendent compte des réceptions et des interactions plurilingues ou qui en recueillent ou mettent en scène.

\section{Remarques conclusives}

31 "Le progrès ne vaut que s'il est partagé par tous " matraquait il y a un temps une publicité de la SNCF. Même si c'est un travail de Sisyphe, nous croyons, comme Louise Dabène, que c'est encore plus vrai et nécessaire pour la didactique du plurilinguisme, et que cette pédagogie de l'alternance des langues, telle que nous l'avons parcourue ici à travers sept scénarios, en offre une gamme d'options pour toutes et tous. Un travail de Sisyphe, certes, car il faut sans cesse chercher, concevoir, réaliser, maintenir, enseigner, intégrer, communiquer, diffuser, publier, démarcher, réseauter, pérenniser. Mais un travail qui devient possible dès lors qu'il s'organise en larges réseaux internationaux comme le promeut depuis 2014 l'APICAD, l'Association pour la Promotion de l'InterCompréhension À Distance ${ }^{13}$, et qu'il prend appui sur de solides travaux comme ceux entrepris autour des référentiels tels que le REFIC, déjà mentionné (De Carlo \& Anquetil, 2019) et le référentiel du projet européen d'évaluation des compétences en intercompréhension EVAL-IC ${ }^{14}$ (Hülsmann et al., 2020). Voici à titre d'exemples, des compétences que peut viser cette pédagogie de l'alternance des langues:

- Savoir accepter les changements de langue et les contacts de langues, chez soi-même, chez l'interlocuteur et chez les autres.

- Savoir questionner les changements de langue (pour le chercheur, pour l'enseignant, pour l'apprenant, pour le grand public).

- Savoir jouer des langues et de la multimodalité oral/écrit/images avec les médias numériques.

- Savoir ne pas tout comprendre (« tolérer l'ambigüité »).

32 Par les projets sur l'Éveil aux langues, comme Evlang, et ceux sur l'intercompréhension comme Galatea, Louise Dabène a cherché à mettre le plurilinguisme à la portée de tous,

- pour valoriser aux yeux de l'école, de la société et des intéressés eux-mêmes, le répertoire plurilingue de ceux qui en ont un ;

- pour montrer aux autres, à celles et ceux qui se considèrent monolingues, que leur environnement, comme tout contexte, est plurilingue, et qu'ils ont tout à y gagner. Par exemple au Brésil où le potentiel des langues amérindiennes est encore peu exploité dans l'éducation ${ }^{15}$, alors même que dans le cadre d'une approche plurielle comme la pratique Érica Sarsur (2020), elles trouveraient toute leur place, à l'instar de ce que proposent Calvo del Olmo \& Felix (2019). Ainsi pourrait-on avoir recours à la richesse que nous offre la toponymie en langue tupi-guarani avec des noms de lieux comme : Itamambuca, Itaú, Itatiaia, Itabira / où le morphème ita signifie pierre, associé aux adjectifs creuse, noire, humide, dressée.

Avec cette finalité, Louise Dabène a lancé, dirigé et tracé des pistes pour les recherches et les développements ultérieurs. Nous espérons avoir montré ici comment ses « héritiers » de l'intercompréhension continuent, en « réseautant » comme elle savait si bien le faire et en fédérant les équipes des projets précurseurs (comme c'est le cas dans Redinter, Miriadi, EVAL-IC, DIPROlinguas, APICAD), à diffuser et développer ses idées. 


\section{BIBLIOGRAPHIE}

Alaoui, D.-C. (2007). Analyse de Comparons nos langues. Alsic [En ligne], Vol. 10, n 2 http:// journals.openedition.org/alsic/681

Alas Martins, S. (2014). A intercompreensão de línguas românicas: proposta propulsora de uma educação plurilíngue. In MOARA, Revista do programa de pos-graduação em Letras.Universidade Federal do Pará. $N^{\circ} 42$, p.117-126, jul./dez. 2014, Estudos Linguísiticos. https://periodicos.ufpa.br/ index.php/moara/article/view/2059

Ali-Bencherif, M. Z. (2009). L'alternance codique arabe dialectal/français dans des conversations bilingues de locuteurs algériens immigrés/non-immigrés. Thèse de doctorat en sciences du langage (option : sociolinguistique), sous la direction de Boumediène Benmoussat et Jacqueline Billiez, Université Abou Bakr BELKAÏD de Tlemcen (Algérie). https://tel.archives-ouvertes.fr/ tel-00496990/document

Albuquerque Costa, H. \& Miranda de Paulo, L. (2012). Français instrumental au Centre de Langues de l'Université de Sao Paulo et intercompréhension : voies possibles pour une refonte de la programmation des cours. In C. Degache et S. Garbarino (Ed.), Actes du colloque IC2012. Intercompréhension : compétences plurielles, corpus, intégration. Université Stendhal Grenoble 3. https://silo.tips/download/1-introduction-resume-resumo

Araújo e Sá, M.-H., Hidalgo Downing, R., Melo-Pfeifer, S., Séré, A. \& Vela Delfa, C. (Ed.) (2009). Intercompreensão em Línguas Românicas: conceitos, práticas, formação. Aveiro : Universidade de Aveiro - CIDTFF - LALE. En ligne sur www.researchgate.net

Araújo e Sá, M-H. \& Melo-Pfeifer, S. (Ed.) (2010). Formação de formadores para a intercompreensão : princípios, práticas e reptos. Universidade de Aveiro - CIDTFF - LALE.

Auger, N. (2004). Comparons nos langues. Démarche d'apprentissage du français auprès d'Enfants Nouvellement Arrivés (ENA). Montpellier : CRDP Languedoc-Roussillon http://asl.univ-montp3.fr/ masterFLE/n.auer/Livret_Comparons.pdf

Auger, N. (2007). Enseignement des langues d'origine et apprentissage du français : vers une pédagogie de l'inclusion. Le français aujourd'hui, 158(3), 76-83. https://doi.org/10.3917/lfa.158.0076

Bégin, C. (2008). Les stratégies d'apprentissage : un cadre de référence simplifié. Revue des sciences de l'éducation, 34 (1), 47-67. https://doi.org/10.7202/018989ar

Blanche-Benveniste, C. (1991). Le projet d'enseignement simultané des langues romanes à l'Université de Provence. Dialogues et Cultures, nº spécial 91-1, p. 9-16

Blanche-Benveniste, C. (1992). Projets d'enseignement et de recherche sur les langues romanes en Europe dans les années 90. Comunicación en ExpoLingua, Madrid.

Blanche-Benveniste, C. ; Valli, A. \& al. (1997). Eurom4 : méthode d'enseignement simultané des langues romanes. Firenze : La Nuova Italia Editrice.

Blanchet, P. (2018). Regard sociolinguistique sur l'Intercompréhension entre langues différentes : enjeux théoriques et pistes sociodidactiques. In S. Garbarino \& C. Degache (Ed.), Intercompréhension en réseau : scénarios, médiations, évaluations, (pp. 28-35). Travaux du CRTT. Université Lumière Lyon 2, IC2014-Projet Miriadi. https://www.miriadi.net/sites/default/files/ ic2014_definitivo_2018_dic_2018.pdf 
Bonvino, E., Caddéo, S., Vilaginés S., Eulàlia \& Pippa, S. (2011). EuRom5. Lire et comprendre 5 langues romanes. www.eurom5.com, Editore Ulrico Hoepli, Milano.

Calvo del Olmo, F. \& Felix, F. (2019). Ñaneñe'ê : Despertar para as línguas autóctones da América Latina. Revue Miriadi, 1 [En ligne], https://publications.miriadi.net:443/index.php?id=202

Díaz, D. \& Campanella, L. (2019). Leitura em português e francês em intercompreensão e o desafio didático de abordar as anáforas. In L. Masello (Éd.), Estudios de Lenguas. Volumen I. Lenguas en la educación superior (pp. 109-128). Montevideo : Mastergraf.

Carrasco Perea, E., Degache, C. \& Pishva, Y. (2008). Intégrer l'intercompréhension à l'université. Les Langues Modernes 1/2008, Revue de l'APLV, 62-74.

Carullo, A. M. \& Torre, M. L. (2009). InterRom : un outil didactique pour l'apprentissage de l'intercompréhension en langues romanes. In P. Moreno (Ed.), Itinéraires et ressources pour le multilinguisme. Synergies Chili n5, 71-90, https://gerflint.fr/Base/Chili5/carullo.pdf

Carullo, A. M., Torre, M. L., Marchiaro, S., Brunel Matias, R., Pérez, A. C., Arróniz, M., Voltarel, Silvina \& Navilli, E. (2007). InterRom. Intercomprensión en Lenguas Romances. Propuesta didáctica para el desarrollo de estrategias de lectura plurilingüe. Dos volúmenes: (1) De similitudes y diferencias ; (2) Hacia el reconocimiento de los esquemas de organización textual. Universidad Nacional de Córdoba Facultad de lenguas. Ediciones del copista.

Chazalon, C., Pérez, M. \& Bras, M. (à paraitre). Une démarche de recherche en Master MEEF $1^{\circ}$ degré : l'identification du verbe en $2^{\circ}$ élémentaire par la comparaison français-espagnol. $R D L C$.

Chaves Da Cunha, J. C. (2001). La bivalence au Brésil : un regard rétrospectif et prospectif. Le français aujourd'hui, 132(1), 54-60. https://doi.org/10.3917/lfa.132.0054

Da Silva, R. C., \& Degache, C. (à paraitre). Romanofonia e Cinema: temas e dinâmicas dos diálogos interculturais em um cenário telecolaborativo em intercompreensão. In F. Calvo del Olmo, C. Degache \& S. Marchiaro (Éds.). Fundamentos, prácticas y estrategias para la didáctica de la Intercomprensión en América Latina. Córdoba: Editorial Facultad de Lenguas.

Dabène, L. (1984). Langues voisines... langues faciles ? In La latinité aujourd'hui, Les Amis de Sèvres, $\mathrm{n}^{\circ} 1$, p. $27-29$

Dabène, L. (1992). Le développement de la conscience métalinguistique : un objectif commun pour l'enseignement de la langue maternelle et des langues étrangères. Repères, 6, 13-21.

Dabène, L. (1994). Le projet européen GALATEA : pour une didactique de l'intercompréhension en langues romanes. Etudes hispaniques $n^{\circ} 22$, Actes du colloque d'Aix-en-Provence, mars 1992, p. 41-45.

Dabène, L. \& Billiez, J. (1988). L'insertion des jeunes issus de l'immigration algérienne. Aspects sociolinguistiques, discursifs et socio-politiques. Rapport de recherche, Centre de Didactique des Langues, Université de Grenoble III.

Dabène, L., Ingelmann, C. (1996). Un multilinguisme en construction : l'éveil de la conscience métalinguistique. Acquisition et interaction en langue étrangère, 7. http://journals.openedition.org/ aile/4914

Dabène, L., López Alonso, C., Séré, A., Martin, E., Le Besnerais, M., Degache, C., Masperi, M., Poulet, M. E., Hédiard, M., Araújo e Sá, M. H. \& Andrade, A. I. (2001). Programme européen d'entraînement à l'intercompréhension entre locuteurs de langues voisines, Projet Galatea. Les Langues Modernes 1/2001 la pluralité linguistique, actes du XXème Congrès mondial FIPLV. Paris, 22-26 juillet 2000, p. 65-71. 
Dabène, O. (2020). L'Amérique latine à l'époque contemporaine. Neuvième édition. U. Paris: Armand Colin.

De Carlo, M. \& Anquetil, M. (2019). Un Référentiel de compétences de communication plurilingue en intercompréhension. REFIC. EL.LE, 8(1), 163-234.

Debyser, F. (1984). Peut-il y avoir une didactique de l'intercompréhension des langues romanes? In La latinité aujourd'hui, Les Amis de Sèvres, $\mathrm{n}^{\circ} 1$, p. 30-35.

Degache, C. (2003). Présentation. Lidil, 28. p. 5-21.

Degache, C. (2018). Quels scénarios pour pratiquer l'intercompréhension interactive ? Entre double mise en abyme, vertige et effectif réduit, l'analyse itérative et contextualisée d'une session spécifique de formation. In S. Garbarino \& C. Degache (Ed.), Intercompréhension en réseau : scénarios, médiations, évaluations, (pp. 39-74). Travaux du CRTT. Université Lumière Lyon 2, IC2014Projet Miriadi. https://hal.archives-ouvertes.fr/hal-02310508

Degache, C., Carrasco Perea, E., Chevalier, C., da Silva, R., Dalençon, A. \& Fonseca, M. (2012). Caractéristiques et formats de l'intégration curriculaire de l'intercompréhension. In C. Degache et S. Garbarino (Ed.), Actes du colloque IC2012. Intercompréhension : compétences plurielles, corpus, intégration. Université Stendhal Grenoble 3 (France), 21-22-23 juin 2012. https://hal.archivesouvertes.fr/LIDILEM/hal-02937434v1

Degache, C. \& Garbarino, S. (2017). Introduction. Jalons, diffusion et itinéraires des approches intercompréhensives. In C. Degache \& S. Garbarino (Ed.), Itinéraires pédagogiques de l'alternance des langues : l'intercompréhension, (pp.7-23). Grenoble : UGA Editions, collection Didaskein. https:// books.openedition.org/ugaeditions/2072

Degache, C. \& Silva, R. C. da (2020). Détours artistiques et pratiques translinguistiques au sein d'un scénario télécollaboratif inspiré par le cinéma. Alsic, [Online], Vol. 23. http:// journals.openedition.org/alsic/4857

Dominguez Fonseca, M. (2017). Didactique du plurilinguisme et intercompréhension intégrée : étude de pratiques en terrain catalan et occitan. Université de Genève. Thèse de doctorat, sous la direction de Laurent Gajo. https://archive-ouverte.unige.ch/unige:96383

Escudé, P. (Ed.) (2008). J'apprends par les langues : manuel européen Euro-Mania : fichier de l'élève : 8-11 ans : conforme au cadre européen commun de référence pour les langues. SCEREN-CRDP Midi-Pyrénées.

Escudé, P. (2012). L'intercompréhension et la triple intégration. Redinter-Intercompreensão, 3, Attraverso le lingue. L'Intercomprensione in ricordo di Claire Blanche-Benveniste. Chamusca, Ed. Cosmos, p. 131-148. http://www.redinter-intercompreensao.eu/numeros-publies/numero-3

Feneuille, J. (1984). Avant-propos. In La latinité aujourd'hui, Les Amis de Sèvres, n¹, p. 3.

Garbarino, S. (2019). Sviluppare competenze in intercomprensione di livello avanzato: il contributo dei descrittori del REFIC. EL.LE, 8(1), 39-64. https://hal.univ-lyon2.fr/hal-02443515/ document

Hawkins, E. (1984). Awareness of Language. An introduction. Cambridge University Press.

Hidalgo Downing, R. (2019). Competencias comunicativas a través de los marcos de referencia de las lenguas. EL.LE, 8(1), 139-160. En ligne sur https://edizionicafoscari.unive.it

Hülsmann, C., Ollivier, C. \& Strasser, M. (2020). Lehr- und Lernkompetenzen für die Interkomprehension: Perspektiven für die mehrsprachige Bildung. (Salzburger Beiträge zur Lehrer/ innen/bildung). Waxmann Verlag. 
Klein, H. G. \& Stegmann, T. D. (2000). EuroComRom - Die sieben Siebe: Romanische Sprachen sofort. Aachen: Shaker Verlag.

Llamas Pombo, E. \& Uzcanga Vivar, I. (1991). Proyecto de enseñanza simultánea de cuatro lenguas románicas : español, francés, italiano y portugués. Europe plurilingue, Revue de l'Assoc. pour le Rayonnement des Langues Européennes (ARLE). Université Paris VIII - St-Denis, p. 8-11.

Masello, L. (2018). Présentation du projet Lectures sur l'Amérique Latine en Intercompréhension Synergies Brésil n 13, p. 109-125. https://gerflint.fr/Base/Bresil13/masello.pdf

Meissner, F.-J., Meissner, C., Klein, Horst G. \& Stegmann, Tilbert D. (2004). EuroComRom. Les sept tamis. Lire les langues romanes dès le début. Aachen : Shaker-Verlag.

McGurn, J. (1991). Comparing languages. English and its European relatives. Awareness of Language. Series Editor Eric Hawkins. Cambridge University Press.

Miranda de Paulo, L. (2018). A Intercompreensão no curso de Letras: formando sujeitos plurilíngues a partir da leitura de textos acadêmicos em línguas românicas. Tese de Doutorado, orientada por Heloisa Albuquerque Costa, Universidade São Paulo (USP-FFLCH). Pós-Graduação em Estudos Linguísticos, Literários e Tradutológicos em Francês, soutenue le 6/11/2018. http:// www.teses.usp.br/teses/disponiveis/8/8146/tde-26032019-145256/pt-br.php

Moore, D. (2001). Une didactique de l'alternance pour mieux apprendre ? Éla. Études de linguistique appliquée, $\mathrm{n}^{\circ}$ 121(1), 71-78. https://doi.org/10.3917/ela.121.0071

Py, B. (1984). À propos d'un noyau de résistance de l'espagnol à son apprentissage par les francophones. Les verbes de déplacement « ir », « venir », « llevar » et « traer ». In La latinité aujourd'hui, Les Amis de Sèvres, nº 1, p. 40-43.

Sarsur Câmara, E. (2020). Abordagens plurais das linguas no ensino fundamental: experiência piloto com pré-adolescentes de uma escola pública de Belo Horizonte. Tese de Doutorado em Estudos Linguísticos (Linguística Aplicada), orientada por Christian Degache, Universidade Federal de Minas Gerais, Faculdade de Letras, POSLIN, soutenue le 9 juillet 2020. https://repositorio.ufmg.br/handle/ $1843 / 33943$

Serrani Infante, S. (2001). Resonancias discursivas y cortesía en prática de lecto-escritura. D.E.L.T.A., 17(1), 31-58. http://repositorio.unicamp.br/bitstream/REPOSIP/202722/1/ S0102-44502001000100002.pdf

Schmitt Jensen, J. (1997). L'expérience danoise et les langues romanes. Le français dans le monde, numéro spécial, janvier 1997, 95-108

Souza, R. E. Gomes de (2013). Didática do plurilinguismo: efeitos da intercompreensão de línguas românicas na compreensão de textos escritos em português. Tese (Doutorado em Linguística Aplicada), orientada por Selma Alas Martins, Univ. Federal do Rio Grande do Norte, Natal. https:// repositorio.ufrn.br/jspui/bitstream/123456789/16388/1/RudsonEGS_TESE.pdf

Tassara, G., Moreno, P. \& Varela, G. (2007). Manual Interlat. Comprensión escrita en portugués, español y francés. Valparaíso, Chile : Ediciones Universitarias de Valparaiso. Présentation sur http:// www.euv.cl/interlat.htm

Villalón, C., Tassara, G. \& Moreno P. (2011). La intercomprensión entre lenguas latinas en las políticas educacionales: el caso de Chile. In D. Álvarez et al. (Ed.), L'intercompréhension et les nouveaux défis pour les langues romanes, (pp.191-210). Agence Universitaire de la Francophonie \& Union Latine. Disponible sur https://www.unilat.org/data/publications/79.pdf 


\section{NOTES}

1. Au moment où nous entrions dans son équipe de recherche.

2. https://www.sciencespo.fr/ceri/fr/cerispire-user/7378/1280

3. Voir par exemple https://dizionari.corriere.it/dizionario_italiano/s/scenario.shtml

4. https://www.aliancafrancesa.com.br/novidades/conheca-o-metodo-de-ensino-hachetteusado-na-alianca-francesa/

5. www.youtube.com/watch?v=_ZlBiAoMTBo

6. www.miriadi.net/diprolinguas, projet mené à bien dans le cadre du Programme de coopération scientifique bilatéral franco-brésilien CAPES/Cofecub. La CAPES (Coordenação de Aperfeiçoamento de Pessoal de Nível Superior) est l'organisme, sous tutelle du Ministère de l'Education nationale brésilien, responsable du $3^{\text {ème }}$ cycle de l'enseignement supérieur et de la formation post-doctorale des enseignants-chercheurs. Le Cofecub est le Comité Français d'Évaluation de la Coopération Universitaire et Scientifique avec le Brésil (www.campusfrance.org/fr/cofecub).

7. À savoir, les langues de scolarisation (portugais et français), les autres langues romanes et les langues présentes dans l'environnement des élèves: langues d'héritage, langues du substrat (amérindiennes, régionales), langues des signes (Libras, LSF), langues des migrants et autres langues additionnelles.

8. Enseignement des langues dans l'état du Minas Gerais pour et par l'intercompréhension, https:// www.miriadi.net/2523-projeto-elmic-minas-gerais. Cette formation, organisée en 2018 et faisant suite à la signature en 2017 d'un accord de coopération entre l'Ambassade de France et le Secrétariat de l'éducation de l'état du Minas Gerais, trouve sa continuité dans les activités du Service de Coopération Linguistique du Consulat Général de France à Rio de Janeiro.

9. Sept cédéroms publiés de 2001 à 2003 (voir liste in Degache, 2003) dont un sur la compréhension orale.

10. www.miriadi.net/5396-2019scuola-ic

11. www.miriadi.net/activity

12. https://www.miriadi.net/6222-romanofonia-e-cinema-4-bambini-partigiani-infanciasclandestinas

13. www.miriadi.net/l-apicad

14. http://evalic.eu/

15. Un objectif de DIPROlinguas, cf. https://www.miriadi.net/diprolinguas/projet-et-sesterrains-d-action

\section{RÉSUMÉS}

Dans un article publié en 1995 dans les ELA, Louise Dabène s'interrogeait sur les conceptions curriculaires qui pouvaient offrir un terrain de déploiement aux pratiques pédagogiques centrées sur l'intercompréhension en langues voisines, notamment pour la famille des langues romanes. $\mathrm{Au}$ vu de la pluralité des réalisations menées à bien depuis 25 ans et de la diversité des fonctions didactiques assumées par l'insertion curriculaire de l'intercompréhension, cet article vise à montrer, à travers un panorama organisé autour de sept scénarios, que les «approches intercompréhensives » ont en réalité la finalité de mettre le plurilinguisme à la portée de tous à travers une pédagogie de l'alternance des langues. Savoir changer de langue, accepter les 
changements de langue et les contacts de langue (chez soi-même et chez l'autre), savoir questionner les changements de langue; voilà quelques unes des compétences qui peuvent être visées par cette pédagogie.

In an article published in 1995 in the ELA, Louise Dabène examined the curricular designs that could best offer a field of deployment for pedagogical practices centered on mutual understanding in languages of the same family, like Romance languages. A wide range of diverse projects centered around the curricular integration of intercomprehension were carried out over the last 25 years. In this article, I focus on seven scenarios to demonstrate that "intercomprehensive approaches in education" strongly contribute to opening access to plurilingualism for all, through a pedagogy of language switching. Knowing how to change languages, accepting language changes and language contacts (at home and with others), knowing how to question language changes; these are some of the skills that can be targeted by this pedagogy.

INDEX

Mots-clés : didactique des langues et du plurilinguisme, intercompréhension, insertion curriculaire, alternance des langues

Keywords : second language teaching and learning, plurilingual education inter-comprehension, language switching in the classroom

\section{AUTEUR}

\section{CHRISTIAN DEGACHE}

Laboratoire LIDILEM, Université Grenoble Alpes (UGA), France et POSLIN Universidade Federal de Minas Gerais (UFMG), Brésil

christian.degache[at]univ-grenoble-alpes.fr

Enseignant-chercheur en didactique des langues, approches plurilingues et TICE, membre du laboratoire LIDILEM (directeur-adjoint de 2010 à 2016). Ses travaux portent sur les stratégies d'apprentissage et les interactions plurilingues dans les environnements numériques autour de scénarios télécollaboratifs pour l'intercompréhension (direction de projets - dont Galanet - et collaborations avec Europe et Amérique Latine, notamment Brésil). Voir CV et publications sur https://cv.archives-ouvertes.fr/christian-degache 